Contents List available at VOLKSON PRESS

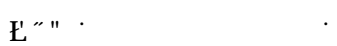

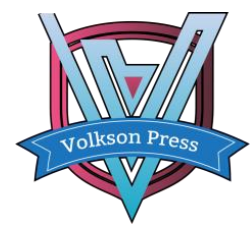

\author{
Intelligent Computing and Information Engineering (ICIE ) \\ DOI : http://doi.org/10.26480/icie.01.2017.99.102 \\ Journal Homepage: : https://www.intelcomp-design.com/
}

\title{
ON REQUIREMENTS OF LOW COST DISTRIBUTED DATA PROCESSING STARTER KIT
}

Kangwon Lee

Korea Polytechnic University, 237 Sankidaehakro Siheung, Kyunggido, Korea

*Corresponding author email: kangwon.lee@kpu.ac.kr

This is an open access article distributed under the Creative Commons Attribution License, which permits unrestricted use, distribution, and reproduction in any medium, provided the original work is properly cited

\section{ARTICLE DETAILS}

\section{Article History:}

Received 12 May2017

Accepted 12 July 2017

Available online 14 September 2017

\section{Keywords:}

Distributed Computing, Low Cost, Low Power, Spatial Footprint, Scalability.

\section{ABSTRACT}

This paper aims to propose a list of requirements for a starter kit of distributed data processing platform intended to give small or medium sized innovative companies: low cost, standalone, ease of use, small footprint, low power, and scalability. The proposed starter kit architecture is composed of a power hub, a network hub and four open source Linux single board computers. For each requirement, this paper also tried to provide with reviews. The cost before tax, shipping and handling can be below USD300. After configuration is done, network connection would not be necessary. (Possibly for data upload/download) Using Linux single board computers would be as easy as using similar desktop computers. The single board computers are roughly the size of a credit card. Total power consumption is expected to be less than 30W. Possible future work includes survey on other experts' opinions and further analysis on performance and thermal managements.

\section{Introduction}

The concept of "Big Data" has been around for a number of years so far. One of the motivations is to make better decisions based on analysis of a great deal of data. However, the "Big" part is often interpreted as "not easy to handle with one computer of the time." Therefore, to process the "Big Data" a multinode network is required. For innovative companies, frequently small or medium sized, it may not be always easy to gain experiences and/or decide to invest on possibly costly data center. Temporarily renting a cloud based service with encryptions is not impossible; however, the business leadership might prefer if it could manage and process some types of sensitive data in-house.

Regarding prior works, some researcher built a cluster of 64 Raspberry Pi Linux computers and evaluated its performance using LINPACK [1,2]. Widriksson and Kim respectively presented possible instructions to build a Hadoop cluster using Raspberry Pi's while Ricart used Beagle Bone Blacks [1-8]. Lee proposed utilizing such network based on the open source for small or medium sized businesses [9].

This paper aims to propose and review a list of requirements for such low cost multi node starter kit for innovative small or medium sized organizations based on open source single board Linux computers such as Raspberry Pi's and Beagle Bone Blacks.

\section{SYSTEM ARCHITECTURE}

\subsection{System Overview}

In author's opinion, following requirements could be considered for a distributed data processing starter kit.

1. Low cost

2. Standalone

3. Ease of use

4. Low spatial footprint

5. Low power consumption

6. Scalable

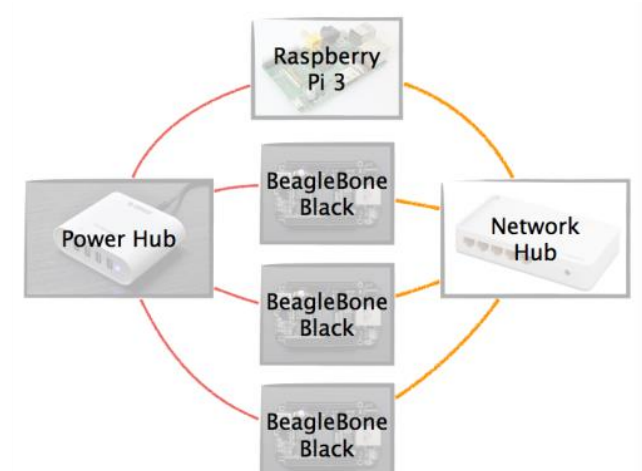

Figure 1: A possible hardware configuration. 
Figure 1 depicts a candidate configuration possibly satisfying majority of the requirements. Each node could run a version of Linux with Java \& Hadoop to implement distributed data processing. Following sections will go through each requirement.

2.2. Cost

Table 1: Expected initial expense for Figure 1.

\begin{tabular}{llll} 
Type & Unit Price [USD] & Quantity [EA] & Cost [USD] \\
\hline Raspberry Pi 3 & 36.89 & 1 & 36.89 \\
\hline BeagleBone Black & 56.69 & 3 & 170.07 \\
\hline 6 port USB power hub & 24.99 & 1 & 24.99 \\
\hline Micro USB cable 3 pack & 7.49 & 0.33 & 2.47 \\
\hline 2.1mm barrel USB cable & 3.95 & 3 & 11.85 \\
\hline 10/100 hub & 9.99 & 1 & 9.99 \\
\hline Ethernet cable 5 pack & 19.99 & 1 & 19.99 \\
\hline
\end{tabular}

Total

276.25

From an online shopping mall, the components of Figure 1 could be purchased at the cost in Table 1 before tax, shipping and handling. To establish a multinode platform using conventional PC's, cost can be more than USD 1000.

\subsection{Standalone}

Once the software is installed and the data is uploaded, connection to another network would not be necessary except code / data update or sending processed result out. Other than these, during the normal operation, no external connection would be normally necessary.

\subsection{Easy to Use}

The proposed system is expected to be based on Linux. To author's knowledge, there are many Linux users and voluntary support structure; hence if introduced to an organization, within a reasonable amount of time, it would be reasonably usable. However, it is expected that additional software could make it even easier to use and understand how it works.

\subsection{Low spatial footprint}

Sometimes a small or medium size organization may not have sufficient space. Especially when located in a high population density area or remnants of past projects are taking up a great deal of the space. It may not be easy to add another PC case in the allocated space.

Both Raspberry Pi and BeagleBone Black are roughly the size of a credit card. If stacked vertically, its foot print could still be similar to the size of a credit card. However, it might be necessary to verify the minimum desired pitch between the PCB's to prevent overheating. The BeagleBone Black can extend its functionalities by attaching a "cape" on top of it; hence no significant problem is expected. Table 2 summarizes the physical dimensions.

Table 2: Physical Dimensions \& Number of USB Ports

\begin{tabular}{|c|c|c|c|c|c|c|}
\hline & & & $w(\mathbf{m m})$ & d (mm) & $\mathbf{h}(\mathbf{m m})$ & USB (EA) \\
\hline \multirow{12}{*}{ RP } & \multirow{2}{*}{ A } & 1 & 85.6 & 56.5 & & 1 \\
\hline & & $1+$ & 65.0 & 56.5 & & 1 \\
\hline & \multirow{5}{*}{ B } & 1 & 85.6 & 56.5 & & 2 \\
\hline & & $1+$ & 85.6 & 56.5 & & 4 \\
\hline & & 2 & 85.6 & 56.5 & & 4 \\
\hline & & 2 ver 1.2 & 85.6 & 56.5 & & 4 \\
\hline & & 3 & 85.6 & 56.5 & 17 & 4 \\
\hline & \multirow{3}{*}{ Compute Module } & 1 & 67.6 & 30 & & 1 \\
\hline & & 3 & 67.6 & 31 & & 1 \\
\hline & & 3 lite & 67.6 & 31 & & 1 \\
\hline & \multirow{2}{*}{ Zero } & PCB ver 1.2 & 67.6 & 31 & & 1 \\
\hline & & PCB ver 1.3 & 65 & 30 & 5 & 1 \\
\hline
\end{tabular}




\begin{tabular}{|c|c|c|c|c|c|c|}
\hline & & & $\mathbf{w}(\mathrm{mm})$ & $\mathrm{d}(\mathrm{mm})$ & $\mathrm{h}(\mathrm{mm})$ & USB (EA) \\
\hline & & W (wireless) & & & & 1 \\
\hline BeagleBone & & & 86.4 & 53.3 & & $1+1$ (mini) \\
\hline BeagleBone & Black & & 86.4 & 53.3 & & $1+1($ mini $)$ \\
\hline
\end{tabular}

2.6. Low power consumption

Table 3: Power Ratings

\begin{tabular}{|c|c|c|c|c|c|}
\hline & & & Power (V) & Current (A) & Power (W) \\
\hline \multirow{13}{*}{$\mathbf{R P i}$} & \multirow{2}{*}{$\mathbf{A}$} & 1 & 5 & 0.3 & 1.5 \\
\hline & & $1+$ & 5 & 0.2 & 1 \\
\hline & \multirow{5}{*}{ B } & 1 & 5 & 0.7 & 3.5 \\
\hline & & $1+$ & 5 & 0.35 & 1.75 \\
\hline & & 2 & 5 & 0.82 & 4.1 \\
\hline & & 2 ver 1.2 & 5 & 0.82 & 4.1 \\
\hline & & 3 & 5 & 1.34 & 6.7 \\
\hline & \multirow{3}{*}{ Compute Module } & 1 & 5 & 0.2 & 1 \\
\hline & & 3 & 5 & 0.7 & 3.5 \\
\hline & & 3 lite & 5 & 0.7 & 3.5 \\
\hline & \multirow{3}{*}{ Zero } & PCB ver 1.2 & 5 & 0.35 & 1.75 \\
\hline & & PCB ver 1.3 & 5 & 0.35 & 1.75 \\
\hline & & W (wireless) & 5 & 0.35 & 1.75 \\
\hline \multicolumn{2}{|l|}{ BeagleBone } & & 5 & 0.5 & 2.5 \\
\hline BeagleBone & Black & & 5 & 0.46 & 2.3 \\
\hline
\end{tabular}

Table 3 shows power ratings of the single board computers. Within in $200 \mathrm{~W}$ power, possibly more than 10 single board computers could be accommodated.

\subsection{Scalable}

The proposed package could let a small or medium size companies evaluate and train on a distributed data processing early-on in house. It would be even better if it can scale to the computational capacities required to tackle applications of more interests.

When more data storage is desired, an external hard disk drive could be added. Table 2 also shows number of USB ports of Raspberry Pi's and BeagleBone Blacks. To extend computational capabilities, one or more (Linux) nodes could be added. Depending on the situation, single board computers such as Raspberry Pi's or BeagleBone Blacks could be added. Or if more powerful node is desired, an off-the-shelf PC box, possibly with Intel CPUs could be considered. However, Amdahl's Law would impose upper limits in the distributed processing power [10].

\section{CONCLUSIONS}

This paper proposed a list of requirements for a distributed data processing starter kit for innovative small or medium sized companies. The proposed system seems to satisfy majority of the requirements under low budget with possibilities of further expansions. Regarding the future work, a survey on innovative experts' opinions on the requirements and further analysis on including but not limited to performance and thermal managements could be pursued.

\section{ACKNOWLEDGEMENT}

This work was supported by Transportation and Logistics Research Project (17TLRP-B117132-02-000000) by Ministry of Land, Infrastructure and Transport of Korea.

\section{REFERENCES}


[1] Cox, S.J., Cox, J.T., Boardman, R.P., Johnston, S.J., Scott, M., O’Brien, N.S. 2014. Iridis-pi : a low-cost, compact demonstration cluster. Cluster Computing, 17 (2), 349-358.

[2] Pi, R. 2017. Wikipedia [online] Available at : https://en.wikipedia.org/wiki/Raspberry_Pi [Accessed 1 Jul. 2017].

[3] Widriksson, J. 2014. Widriksson.com. 2310 2014. [Online]. Available: http://www.widriksson.com/raspberry-pi-hadoop-cluster/. [Access : 274 2017].

[4] Kim, J. 2016. Raspberry Pi Hadoop Cluster - 1. 165 2016. [Online]. Available: http://jeongchul.tistory.com/489. [Access : 284 2017]. (In Korean)

[5] Welcome to Apache ${ }^{\mathrm{TM}}$ Hadoop $®$ ! 2017. Apache Foundation, Online. Available: http://hadoop.apache.org/index.pdf [Access: 0807 2017].

[6] Zikopoulos, P., Eaton, C. 2011. Understanding Big Data : Analytics for Enterprise Class Hadoop and Streaming Data (1st ed.). McGraw-Hill Osborne Media.

[7] Ricart, D. 2013. "Linux.com," 2111 2013. [Online]. Available: https://www.linux.com/blog/setting-multi-node-hadoop-cluster-beagle-bone-black. [Access : 274 2017].

[8] BeagleBoard. 2017. Wikipedia [online] Available at: https://en.wikipedia.org/wiki/BeagleBoard\#BeagleBone_Black [Accessed 1 Jul. 2017].

[9] Lee, K. 2017. How to Build a Distributed Data Processing Platform on a Network of Single Board Open Source Linux Computers. KSME IT Convergence Division Spring Conference, Ulsan, Korea, May 2017.

[10] Amdahl's law. 2017. Wikipedia [Online]. Available: https://en.wikipedia.org/wiki/Amdahl\%27s_law. [Access : 08072017 ]. 